\title{
Implementación de un repositorio para apoyo a la gestión administrativa
}

Implementation of a repository to support administrative management

\section{Maria E. Sánchez}

Universidad Nacional del Nordeste. Facultad de Ciencias

Exactas y Naturales y Agrimensura, Argentina

ely_san_barr@hotmail.com

(iD https://orcid.org/0000-0003-2942-0116

\author{
Sonia I. Mariño \\ Universidad Nacional del Nordeste. Facultad de Ciencias \\ Exactas y Naturales y Agrimensura, Argentina \\ simarinio@yahoo.com \\ (DD https://orcid.org/0000-0003-3529-7003
}

\section{ReSUMEN:}

La información es el activo intangible más importante del cual dispone la gestión administrativa. El artículo se centra en la implementación de un repositorio de documentos digitales administrativos. Se describe el método seguido para su desarrollo, implementación y validación en un contexto universitario, que consta de cuatro fases. Particularmente, la fase 3 trata la construcción del repositorio administrativo de acceso abierto para capturar, manejar, guardar y conservar los documentos digitales, se seleccionó como herramienta informática un Manejador de Contenido Empresarial, en este caso Nuxeo. Los resultados de la implementación permitieron a la Dirección General de Liquidación de Haberes de la Universidad Nacional del Nordeste gestionar la información producida y agilizar la automatización de recepción de las novedades necesarias para las liquidaciones de haberes. Dicho repositorio constituyó una herramienta eficaz para la gestión de información brindando a la universidad una solución integral para la gestión, tramitación y resguardo de los documentos digitales, agilizando la búsqueda y recuperación de la información. Los logros sugieren la mejora de procedimientos mediados por TIC y se constituyen en un aporte al área de repositorios digitales en contextos administrativos.

Palabras CLAVE: Gestión administrativa, Gestión de la información, Repositorio administrativo, Universidades, Argentina.

\section{Abstract:}

Information is the most important intangible asset that has administrative management. The article focuses on the implementation of a repository of administrative digital documents. The method followed for its development, implementation and validation in a university context is described, which consists of four phases. Particularly, Phase 3 deals with the construction of the open access administrative repository to capture, manage, save and preserve digital documents, it was selected as a computer tool and Business Content Manager, in this case Nuxeo. The results of the implementation allowed the General Directorate of Settlement of Assets of the Universidad Nacional del Nordeste to manage the information produced and speed up the automation of reception of the news necessary for the settlements of assets. This repository is an effective tool for information management, providing the university with a comprehensive solution for the management, processing and safeguarding of digital documents, speeding up the search and retrieval of information. The achievements suggest the improvement of intermediate ICT procedures and constitute a contribution to the area of digital repositories in administrative contexts.

KEYWORDS: Administrative management, Information management, Administrative repository, University, Argentina.

\section{INTRODUCCIÓN}

Las universidades se encuentran en un proceso continuo de cambios generados e incrementados por la información y el conocimiento. Así, las herramientas de tecnologías de la información y comunicación (TIC) 
y la gestión del conocimiento (GC) interactúan como un mecanismo de acción para el análisis de la información física y digital producida en diversas áreas.

El artículo describe la estrategia y los resultados preliminares logrados al implementar un repositorio digital administrativo en un contexto universitario específico como es la Dirección General de Liquidación de Haberes (DGLH) de la Universidad Nacional del Nordeste (UNNE). Mediante la herramienta Nuxeo, con el fin de permitir capturar, manejar, guardar y conservar los documentos digitales para producir información vital para ejecutar las consultas y la toma de decisiones en la gestión universitaria.

En este contexto de innovación mediada por TIC, los repositorios se identifican como una de las estrategias del acceso abierto (AA) que promueven la modernización.

De Giusti (2020) define AA como la disponibilidad libre y gratuita de la literatura científica en Internet, que incluye permisos de lectura, descarga, copia, impresión, distribución o cualquier otro uso legal, con la única condición de que los autores mantengan su control sobre la integridad de la obra y que su autoría sea reconocida.

Además, De Giusti (2014) expone una metodología de evaluación para repositorios institucionales (RI). En Doria, Del Prado \& Haustein (2015) se aborda el uso de los repositorios digitales y software open source en el contexto universitario. Molina Piñeiro, Marrero Sera \& Puente Puentes (2015) proponen RI orientados al acceso y visibilidad de la producción científica y académica. Por su parte, Fushimi (2018) trata la "evolución y el proceso de construcción de los repositorios digitales institucionales" en el contexto universitario nacional argentino en el período 2008-2015. Andrés, Amadio, Wursten, \& Legaria (2020) proceden a realizar un análisis de un RI en una universidad argentina en el período 2015-2019 desde el desarrollo socio-tecnológico.

La reseña previa ilustra la relevancia de tratar el diseño y la implementación de RI en consonancia con los procesos de modernización e innovación organizacional. Además, los antecedentes muestran el elevado número de iniciativas orientadas a la promoción de las publicaciones científicas-tecnológicas y académicas, identificándose un nicho en el abordaje de repositorios de soporte a los procesos administrativos.

La información es el activo más importante en el contexto administrativo de la universidad, se han ideados diferentes opciones para la búsqueda y la transmisión de información, para el acceso y las políticas de preservación, entre otras cuestiones relacionadas. Una de las soluciones, es definir un repositorio digital, que permita el acceso a contenidos y documentos administrativos.

Porro \& Gálvez (2018, p. 3) analizan algunas definiciones en torno a un RI, y establecen que se trata de "un espacio digital que permite organizar, almacenar y recuperar información de alto valor”, igualmente concede el otorgamiento de visibilidad a los trabajos científicos realizados por los miembros del centro, por lo que, da la posibilidad de preservar la memoria intelectual del mismo.

En el mundo digital, Polanco-Cortés $(2014$, p. 3) define a un repositorio digital como "un medio para gestionar, almacenar, preservar, difundir y facilitar el acceso a los objetos digitales que alberga”.

Asimismo, De Giusti (2019, p. 2) conceptualiza a los repositorios como "archivos digitales provistos de un conjunto de servicios web centralizados, creados para organizar, gestionar, preservar y ofrecer acceso libre a la producción científica, académica o de cualquier otra naturaleza cultural, en soporte digital, generada por los miembros de una organización".

Los repositorios deben tener una política definida y asegurar:

- El autoarchivo, es decir, cualquier usuario registrado podrá subir un documento/información, que luego será catalogado y depositado en la colección que corresponda.

- La interoperabilidad entre sistemas de diferente naturaleza.

- El libre acceso a los documentos y, así, a la información

- La preservación a largo plazo de los documentos, según las políticas definidas por la universidad. 
El éxito del movimiento de AA (Fushimi, 2016) ha dado como resultado una creciente cantidad de repositorios de diferente índole. Estos pueden agruparse de acuerdo a diversos criterios como menciona De Giusti (2014) y se encuentran resumidos en la Figura 1:

- RI: almacenan, preservan, diseminan y dan acceso a la producción intelectual de los miembros de una institución, pueden contener solamente la producción intelectual/científica, o reunir también colecciones especiales que son bibliotecas digitales (documentación administrativa, etc.).

- Repositorios temáticos: reúnen, preservan y dan acceso a contenidos de una disciplina o área temática. Pueden ser creados y mantenidos por instituciones académicas o de investigación, o bien por organismos gubernamentales.

- Repositorios de datos: almacenan y preservan los datos científicos generados en el proceso de investigación o existen como repositorios independientes, pero también pueden estar integrados en RI.

- Repositorios huérfanos: permiten el archivo de trabajo de autores que no tienen acceso a otro repositorio (institucional o temático). En general, son establecidos a nivel nacional.

FIGURA 1

Tipos y ejemplos de repositorios.

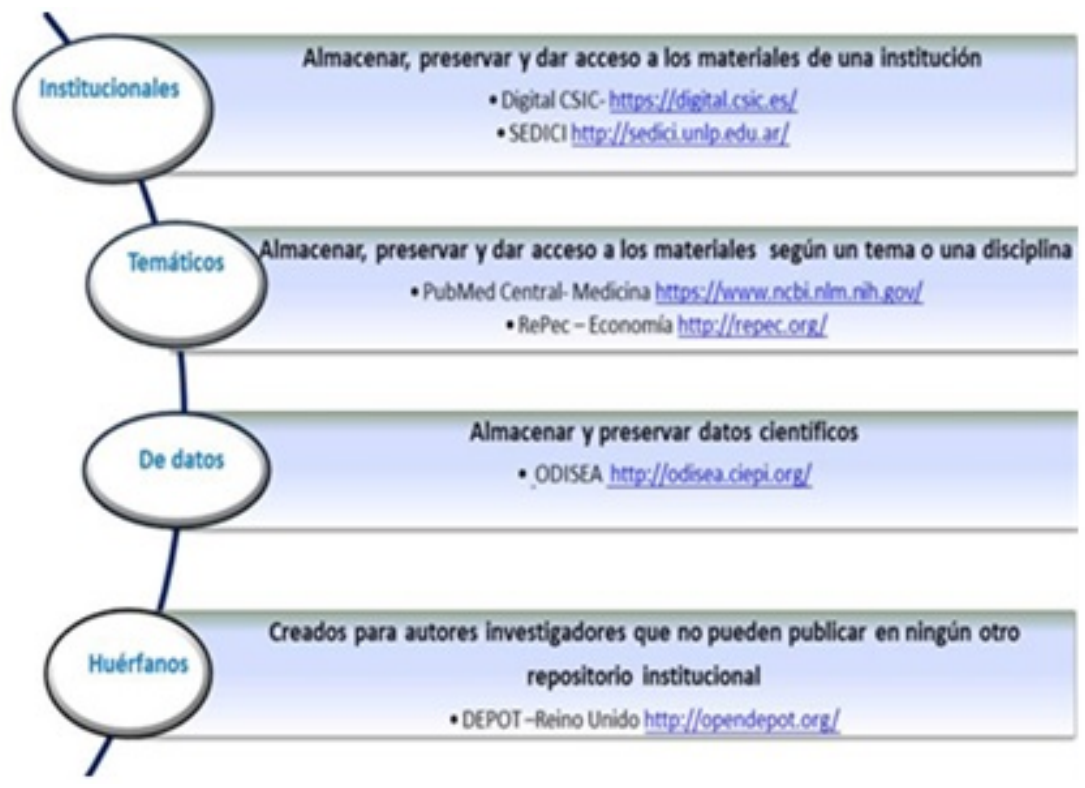

Fuente: elaboración propia

A partir de aquí, el presente trabajo se organiza en cuatro apartados. Inicialmente se sintetiza el marco teórico en que se sustenta la propuesta. A continuación, se presenta la metodología seguida. En los resultados, se describe la implementación del repositorio digital, y, finalmente, se expresan las conclusiones.

\section{El movimiento abierto en Argentina. Ley 26.899}

El acceso a la información a través de RI se ve favorecido por las iniciativas que propone el AA. Por ello, recoge, almacena y da acceso a resultados como publicaciones y/o documentos digitales.

En Argentina se sentaron las bases del movimiento de AA en noviembre del año 2013, cuando se sancionó la Ley 26.899 de creación de Repositorios Digitales Institucionales de AA, ya sean propios o compartidos. Esta disposición fuerza a las instituciones educativas a crear repositorios de AA (Nakano y Azrilevich, 2017): 
... en los que se depositará la producción cientifico tecnológica resultante del trabajo, formación y/o proyectos, financiados total o parcialmente con fondos públicos, de sus investigadores, tecnólogos, docentes, becarios de posdoctorado y estudiantes de maestría y doctorado. Esta producción cientifico-tecnológica abarcará al conjunto de documentos (articulos de revistas, trabajos técnicos cientificos, tesis académicas, entre otros), que sean resultado de la realización de actividades de investigación... (Art. $1^{\circ}$ Ley 26.899/2013).

Los RI accesibles a través de internet, se convirtieron en una herramienta estratégica para asegurar que las publicaciones científicas y académicas de las universidades puedan recopilar, catalogar, acceder, gestionar, difundir y preservar en el tiempo. El Sistema Nacional de Repositorios Digitales (SNRD MINCYT), tiene como objetivo disponer a la comunidad científica y los ciudadanos el acceso a los resultados de la investigación financiada por el estado nacional.

Aun cuando existen numerosos repositorios, especialmente dedicados al resguardo y difusión de las producciones científicas-académicas (Molina Piñeiro, Marrero Sera \& Puente Puentes, 2015), se detectó la necesidad de disponer de un repositorio administrativo, para apoyar la gestión inteligente y la toma de decisiones oportuna. La UNNE dispone de un RI identificado como RIUNNE. En este espacio se almacenan las publicaciones académicas, científicas y técnicas realizadas por los docentes, investigadores y alumnos de posgrado de la universidad, sin embargo, carece de un repositorio administrativo. Con el propósito de cubrir esta falencia, se presenta a continuación la propuesta, considerada innovadora y necesaria en el contexto de la organización.

\subsection{Herramientas para manejar contenido empresarial: Nuxeo}

En las últimas décadas, se han producido avances importantes en tecnologías para la generación, la transformación y la distribución de la información. Uno de ellos se corresponde con herramientas parametrizables para la gestión empresarial.

Un manejador de contenido empresarial o Enterprise Content Management (ECM) se define como una herramienta informática destinada a la administración, uso y almacenamiento de la información electrónica independientemente de su ubicación, formato y tiempo, es un desafío hoy en la actualidad (Mendoza Espinoza, 2012, p. 34).

Un manejador de contenido empresarial es "un conjunto de tecnologías, herramientas y métodos utilizados para capturar, categorizar, manejar, guardar, conservar y entregar contenido a través de una empresa" (Kampffmeyer, 2006). Además, brinda avances importantes en métodos y tecnologías para el manejo de la información de manera eficiente utilizando el conocimiento de la institución, y proporcionando procesos, según sea necesario (Eíto-Brun, 2014; Kampffmeyer, 2006). Por ello, se las considera herramientas orientadas a promover los programas de gestión del cambio organizacional, tal como sostienen Mendes \& $\operatorname{Bax}(2018)$.

Además, un ECM ofrece una infraestructura para las nuevas tecnologías de información basadas en entornos web y se constituye como repositorio único para todos los tipos de información. Se usa como un almacén de contenido que combina la información de la institución en un repositorio que posee una estructura singular. Todas las aplicaciones envían su contenido a un sola plataforma, que, a su vez, provee la información necesaria a todas las aplicaciones.

Según Kampffmeyer (2006), los cinco componentes principales que posee un ECM son:

- Componente de captura: creación de información mediante la conversión de documentos en papel a formatos electrónicos. En este caso, por medio de un dispositivo como es el escáner se pueden transformar los documentos en papel a formato digital.

- Componente de manejo: conecta, modifica y emplea información a través de medios electrónicos, asistido por software. 
- Componente de almacenamiento: respalda la información que cambia frecuentemente.

- Componente de Entrega: proporciona a los usuarios finales la información solicitada

- Componente de preservación: realiza copias de seguridad.

Nuxeo es una potente herramienta de ECM y gestión documental de código abierto, seleccionada por el Sistema de Información Universitaria (SIU) en la Argentina (Nuxeo, s. f.). La UNNE integra el consorcio SIU por lo cual se considera ideal su selección y parametrización. Entre los beneficios de Nuxeo se pueden mencionar los siguientes:

- Es un sistema de código abierto, con una arquitectura robusta, de bajo coste y fácil de usar.

- Ofrece una gestión de documentos usando una interfaz familiar lo cual provee facilidad de interacción con el usuario.

- Brinda un sistema de almacenamiento de los documentos, su seguridad es muy confiable y estable, además de que para implementar la seguridad posee gestión robusta de usuarios, grupos y roles.

- Organiza y facilita la gestión de contenidos de todo tipo de documentos ofimáticos, presentaciones, imágenes, .xml, multimedia etc.

- Permite la gestión del ciclo de vida de los contenidos: crear, compartir, versionar, aprobar, publicar, retirar, archivar.

- Dispone de herramientas como foros, notificaciones, RSS, blogs, wiki, entre otras, para el trabajo colaborativo.

- Resulta altamente escalable, disponible y extensible.

\section{Metodología}

El método para lograr los resultados presentados en este trabajo se centra en un abordaje descriptivo asociado a la elaboración de un procedimiento diseñado ad-hoc que incluye las fases que se presentan a continuación. El estudio de caso se realizó entre los años 2018-2020.

Fase 1. Revisión sistemática de la literatura. Especialmente orientada a relevar información sobre repositorios que contribuya a su implementación. Aspectos preliminares en este sentido se presentaron en un trabajo precedente (Sánchez \& Mariño, 2020).

Fase 2. Contextualización de la propuesta. Se eligió como objeto de indagación y validación la DGLH-UNNE. Con fines de acotar la problemática objeto de interés, dirigida a mejorar los procesos de documentación digital de los haberes de los agentes de la universidad, se procedió a diagnosticar las áreas que integran el proceso de liquidación de sueldos. Para ello, se seleccionaron cuestionarios y encuestas como técnicas e instrumentos de recolección de información. El objetivo que guió la construcción de estos instrumentos se sustentó en la necesidad de conocer los documentos físicos-digitales, su preservación y los recursos disponibles que se integran a este proceso mensual de relevancia administrativa-financiera. En Sánchez, Bózzola, Soler \& Mariño (2020) se describen los resultados de esta implementación que sustentó una parte esencial de los resultados que se presentan en este artículo.

Fase 3. Definición de directrices para la implementación del repositorio de documentos administrativos. Se revisaron las leyes de datos personales, el habeas data en Argentina por la Ley 25.326 (2000) y la normativa de la UNNE. Además, se consideraron y adaptaron los lineamientos establecidos en el manual LEADIRS II para implementar los repositorios (Barton \& Water, 2004-2005). Este manual es un suplemento y ofrece consejos prácticos, así como hojas de trabajo que se pueden usar para empezar su propio programa de repositorios y las directrices para la creación de RI, descriptas en Bustos González y Fernández Porcel (2008): 
- Identificar y seleccionar temas inherentes al objeto de estudio, mediante la lectura, examen y experimentación de la bibliografía seleccionada

- Diseñar un plan de servicio: consistente en las siguientes actividades:

- Realizar una evaluación de las necesidades de la Universidad. En Sánchez et al. (2020) se describieron los hallazgos que sustenta la presente propuesta.

- Crear una planificación, concerniente al diseño e implementación del repositorio.

- Desarrollar políticas de actuación que gestionen la recopilación de contenidos, su distribución y mantenimiento.

- Formar el equipo: interno y externo. Lo expuesto demandó la constitución de un equipo de trabajo con agentes de la DGLH, que afrontó la definición e implementación tecnológica del repositorio, considerando las normativas asociadas. El equipo externo se constituyó con los representantes de las áreas de liquidaciones de las distintas unidades académicas e institutos dependientes de la UNNE.

- Identificar y determinar la tecnología requerida, que comprende: elegir, instalar, configurar y parametrizar el software ECM.

- Poner en funcionamiento el repositorio.

- Difundir el servicio del mismo.

Fase 4. Implementación y validación en un contexto determinado. A efectos de comprobar la propuesta, se seleccionó un área administrativa de la organización universitaria. En este caso, la DGLH-UNNE.

\section{Resultados}

La DGLH-UNNE, recibe mensualmente un gran número de documentos físicos (en formato impreso) referente a las novedades de los agentes en relación de dependencia pertenecientes a las 24 unidades académicas/institutos (en adelante UA/I) ubicadas en las provincias de Corrientes y Chaco. Los directores de personal de cada UA/I incorporan esta información en el sistema de gestión de Recursos Humanos SIU Mapuche, y los expertos de la DGLH controlan e interpretan la documentación con un listado de apoyo de las novedades que genera el mencionado sistema SIU. Cabe aclarar que el sistema SIU-Mapuche ejecuta integralmente la gestión de recursos humanos universitaria. Además, mantiene el legajo del empleado actualizado y constituye una base para obtener información útil para la organización. Se basa en un legajo electrónico único, que es la fuente de información para la gestión de personal, teniendo en cuenta los cambios en la legislación laboral vigente.

Al finalizar el mes, se envía dicha documentación a la Dirección de Legajos (DL) para archivar en el legajo de cada agente. El elevado volumen de documentos refleja un método de archivo lento y que genera un cúmulo de información que requiere el espacio físico adecuado para su almacenamiento.

Por lo expuesto, se optó por la GC como estrategia para impulsar el cambio y hacer frente a la innovación. Para lograrlo, se vincularon las herramientas de TIC con la GC, para fortalecer la gestión administrativa orientada a la toma de decisiones.

La exploración se llevó a cabo desde la perspectiva del conocimiento tácito y explícito basándose en el modelo de GC de Nonaka y Takeuchi (1995), como mecanismo de acción para el análisis de la información.

A través de la definición de técnicas e instrumentos de recolección de información se identificó el estado actual de la información disponible en formato impreso y digital, tal como se detalla en Sánchez et al. (2020).

Se aplicaron encuestas y entrevistas para entender desde la perspectiva del entrevistado y de su experiencia con relación al contexto real de actuación. El universo encuestado estuvo constituido por directores en personal de cada UA/I (24), operadores/auxiliares de liquidación de haberes de cada UA/I (20) y expertos en la recepción de novedades de la DGLH (4). Se estableció como propósito explorar, conocer y analizar los documentos y las normativas contempladas en sus actividades diarias (Sánchez et al., 2020). 
La encuesta, como método empírico, permitió recopilar información de personas para describir, comparar o explicar su conocimiento, sus actitudes o su comportamiento, siguiendo lo expuesto por Kitchenham \& Pfleeger (2008) y Género Bocco, Cruz-Lemus \& Piattini Velthuis (2014). También se capturó el conocimiento existente, disperso y el capital intelectual útil referido a las áreas implicadas en el estudio, con el propósito de determinar su estructura organizacional, los tipos de información y documentación recibida y producida.

En la Figura 2 se presentan los pasos seguidos para la creación del repositorio de documentos administrativos de la UNNE, que corresponden a la Fase 4 de la secuencia metodológica explicada precedentemente.

FIGURA 2

Pasos para la creación de un repositorio

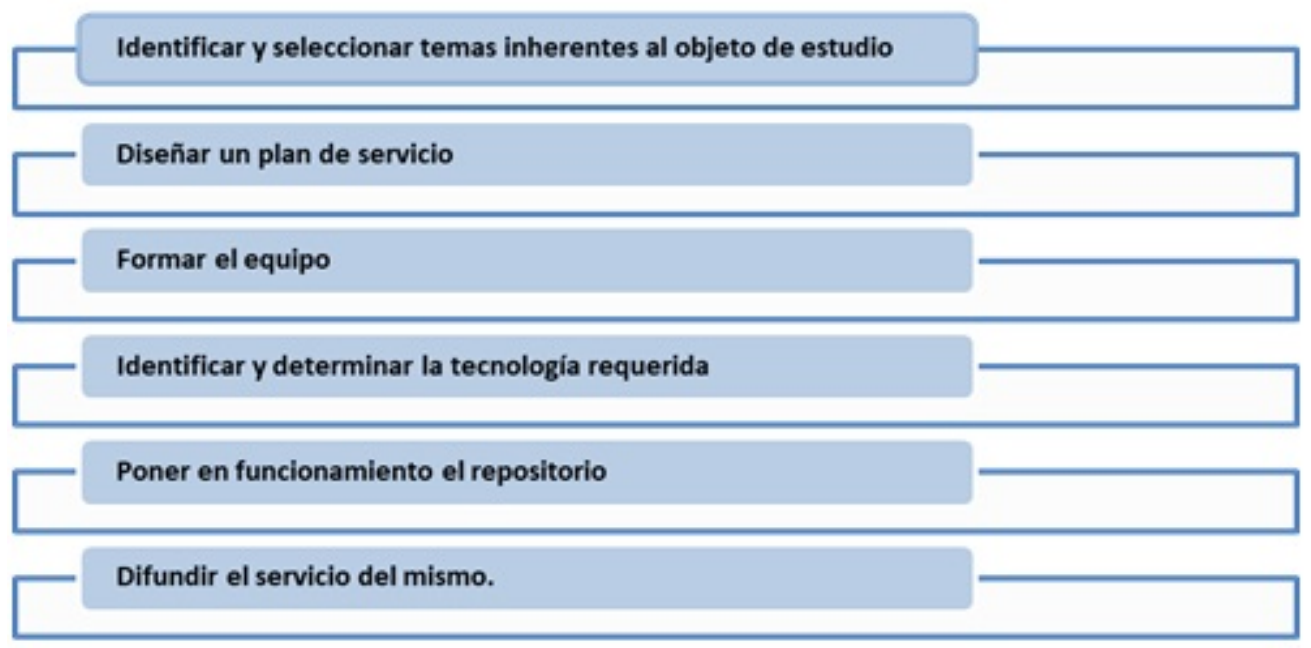

Fuente: elaboración propia.

Las actividades desarrolladas para la creación del repositorio fueron: análisis de requerimientos, diseño y selección de la tecnología, que a su vez implicó la conformación de un equipo interno quienes definieron, instalaron y configuraron el repositorio.

Los requerimientos de hardware y de software para el uso adecuado y eficiente incluyeron la definición de:

- Almacenamiento de una base de datos documentales (BDD): en ellas se hace uso de metadatos (palabras clave y/o descriptores), considerando a la base de datos como un conjunto de identificadores y documentos, y los documentos a su vez como un conjunto de campos y valores. Las BDD al no contar con una estructura estricta las hace más eficientes al momento de manejar grandes volúmenes de documentos. Y la ausencia de relaciones, facilita la replicación de datos.

- Hardware : en él se contemplaron:

- Escáneres y dispositivos de digitalización: los documentos originales, una vez preparados, son transformados en documentos digitales, los cuales serán guardados o almacenados en BDD.

- Servidores: contienen la información digitalizada. Los usuarios se conectarán a un servidor para acceder a dicha información, para su modificación o revisión.

- Software: en él se consideraron herramientas ECM Open Source, en particular, Nuxeo, base de datos PostgreSQL y la aplicación web que integra el repositorio con el sistema SIU Mapuche a través del Sistema de Digitalización (SD-DGLH). 
- Redes: permitieron a los usuarios el acceso a la información disponible en los repositorios. Se contemplaron las redes locales, existentes en la universidad.

- Usuarios: a través de la interfaz del usuario, se accede al sistema de digitalización (SD-DGLH) y por este medio a los documentos digitalizados. Es posible realizar las modificaciones o consultas a los mismos de acuerdo a los niveles de seguridad asignados a los perfiles de usuarios registrados en el sistema, que pueden asumir el rol de: administrador, operador, consulta e invitado.

En referencia a la implementación, el repositorio Nuxeo se integra con el sistema SIU Mapuche a través de un Sistema de Digitalización (SD-DGLH) centralizado en la DGLH de la UNNE, cuyo funcionamiento se inició en el año 2019.

La conformación del Sistema de Gestión Documental (SGD) para la digitalización está compuesta por:

- Un repositorio único Nuxeo configurado para almacenar, organizar y gestionar los documentos internos de la institución previamente digitalizados. Objeto de descripción en el presente artículo.

- El Sistema de Digitalización en la DGLH (SD-DGLH), que brinda una interfaz de usuario web, para establecer las acciones definidas y relativas con las operaciones de los documentos: ingreso, asignación de tareas y búsqueda, como se mencionó previamente.

- Servicios web, que permiten la comunicación entre la interfaz web y el repositorio Nuxeo. Es decir, se aplicó la arquitectura Simple Object Access Protocol (SOAP) (Blancarte, s.f.) para el funcionamiento del sistema informático. SOAP es un protocolo basado en $X M L$ de W3C para intercambiar información a través de HTTP, provee un método simple para el intercambio de mensajes entre aplicaciones. Los servicios web usan SOAP para el envío de mensajes entre servicio y cliente.

En la Figura 3 se ilustra la arquitectura del SGD para la digitalización, que fue propuesto e integrado por Nuxeo, SIU Mapuche y SD-DGLH. 
FIGURA 3

Arquitectura del Sistema de Gestión Documental

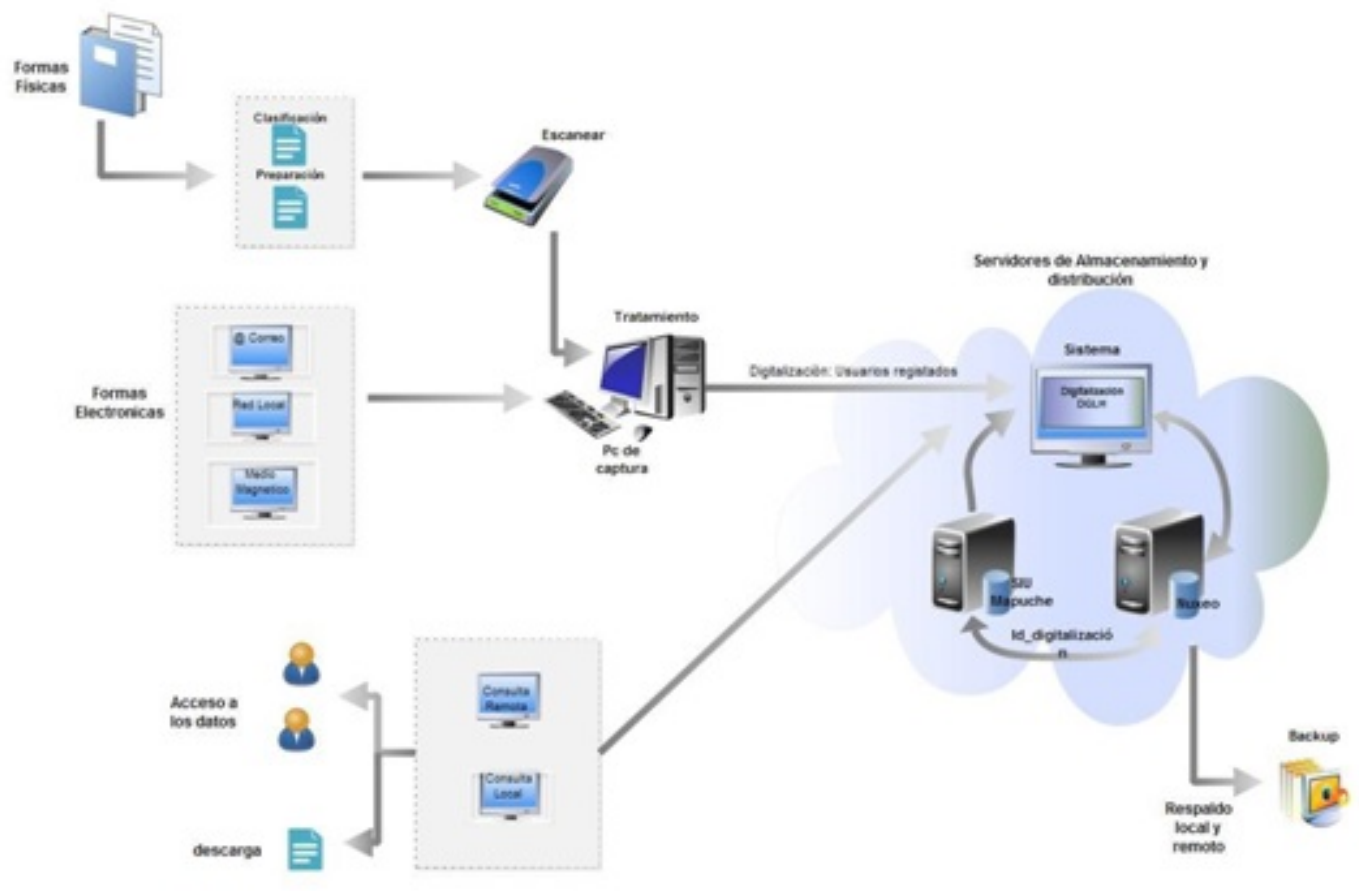

Fuente: elaboración propia.

Luego de que concluyó el diseño del sistema informático SD-DGLH, a través del cual se capturan, almacenan y procesan los documentos, se procedió a su implementación. Cabe aclarar que esta actividad, implicó el compromiso con las distintas áreas de gestión administrativa vinculadas a la liquidación de haberes de la universidad.

En la Figura 4 se muestra la variabilidad de la digitalización de los documentos entre los meses de febrero de 2018 a marzo de 2020. La gráfica representa la implementación del procedimiento y el efectivo uso del repositorio en el contexto universitario de referencia. Evidencias que reflejan los logros derivados de la aplicación del procedimiento y repositorio propuesto en este artículo. Los ejes de las " $\mathrm{x}$ " representan los meses del año y en los ejes de las "y" la cantidad de documentos digitalizados. 
FIGURA 4

Documentos digitalizados por año

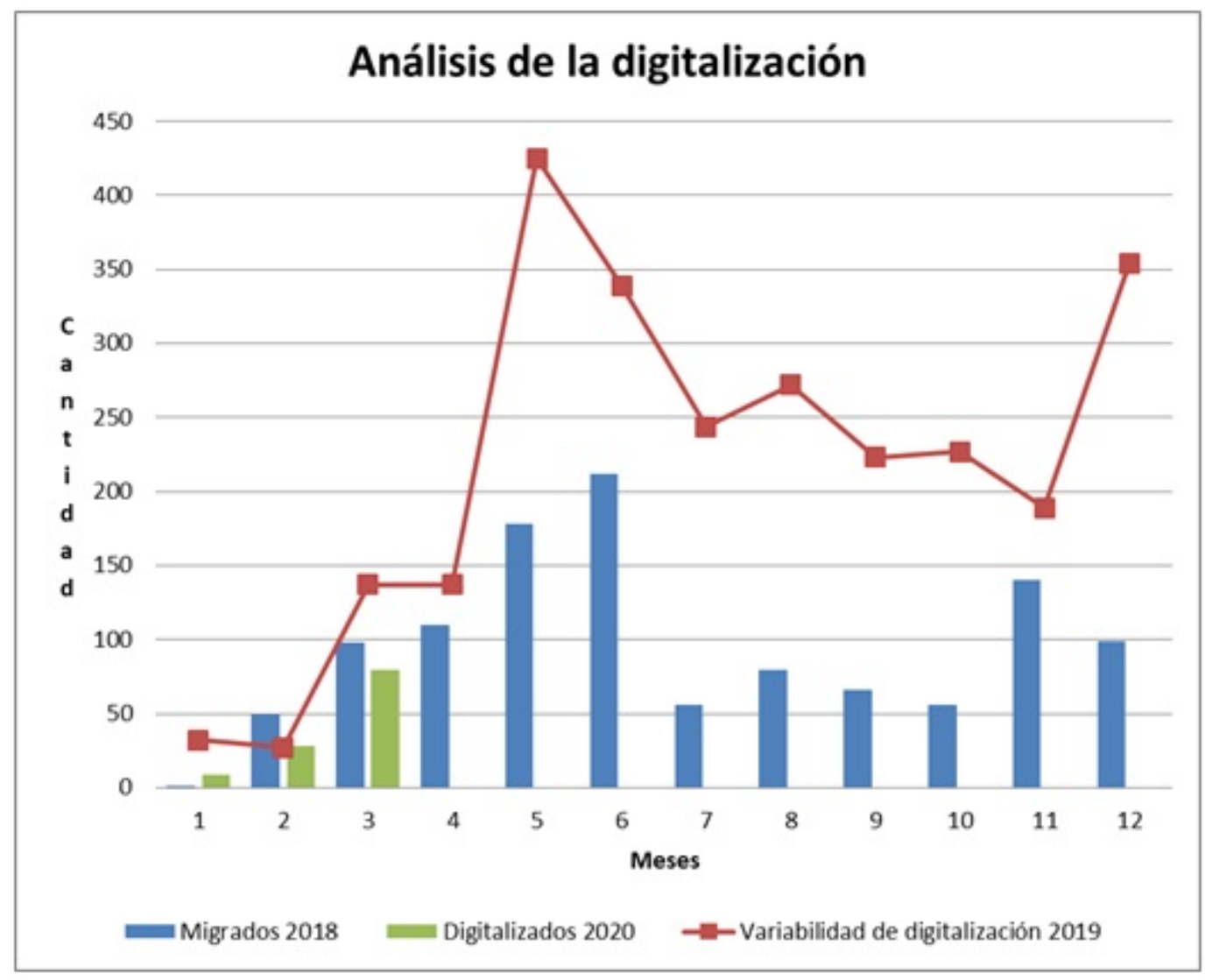

Fuente: elaboración propia.

Cabe destacar que la adopción del procedimiento y del efectivo uso del repositorio es producto de actividades de capacitación y sensibilizaciones realizadas en el mes de mayo de 2019, dirigidas al sector de liquidación de haberes de las unidades académicas e institutos que conforman la UNNE. Estas actividades están comprendidas en las fases denominadas: desarrollar políticas de actuación que gestionen la recopilación de contenidos, su distribución y mantenimiento, y así poder difundir el servicio mencionadas en la fase 4 del método descripto.

\section{Conclusiones}

El artículo expone una solución implementada en una universidad pública nacional orientada a apoyar la captura, el almacenamiento, el procesamiento y el despliegue de documentos administrativos, y validada en el área de liquidación de haberes plasmada en una lógica de ejecución e implementación de un repositorio administrativo. Este recurso aporta a la modernización institucional cubriendo una carencia previa para gestionar la información y aportar a la toma de decisiones.

En su definición, se consideraron las redes de colaboración explícitas a la que se integra la universidad como el consorcio SIU, las leyes nacionales sobre el movimiento abierto, la ley de protección de datos personales y determinados aspectos tecnológicos mencionados en el artículo. Además, se deben destacar, las redes internas implícitas de colaboración, que emergen, se consolidan y evidencian en el compromiso asumido por sus actores de las unidades académicas e institutos al adoptar el procedimiento y continuamente contribuir con datos de alto valor para la toma de decisiones. 
Es así como se evidencia la adopción y promoción del trabajo colaborativo en red en dos sentidos. Uno de ellos implicó adoptar un repositorio open source como es Nuxeo, dada su recomendación por el consorcio SIU al que se integra la universidad. En el otro sentido, se referencia a la implementación del procedimiento y el repositorio en el contexto de la universidad, lo mencionado sostiene en las evidencias que aseguran un uso continuo,

Por lo expuesto en párrafos precedentes, es de destacar la sinergia de esfuerzos en las áreas gerenciales, administrativas y técnicas, para aportar a procesos de GC y toma de decisiones en un camino hacia la modernización de las organizaciones.

Por lo descripto, este artículo constituye un aporte al área de repositorios digitales, especialmente en el ámbito administrativo de universidades, siendo un modelo para gestionar y preservar sus documentos orientado a diversas áreas de la institución u otras organizaciones.

\section{REFERENCIAS}

Andrés, G., Amadio, C. C., Wursten, A. \& Legaria, J. I. (2020, junio 1). Repositorios institucionales digitales de acceso abierto: una mirada socio-tecnológica. Estudio de caso del repositorio de la Universidad Nacional de Entre Ríos Argentina. DOI: https://doi.org/10.34096/ics.i42.7404

Barton, M. R. y Waters, M. M. (2004-2005). Cómo crear un repositorio institucional. Manual LEADIRS II. Recuperado de https://www.recolecta.fecyt.es/sites/default/files/contenido/documentos/mit.pdf

Blancarte, O. (s. f.). SOAP vs REST ¿cuál es mejor? Software architecture. Recuperado de https://www.oscarblancarte blog.com/2017/03/06/soap-vs-rest-2/

Bustos González, A. \& Fernández Porcel, A. (2008). Directrices para la creación de repositorios institucionales en universidades y organizaciones de educación superior. Chile. Recuperado de https://repository.urosario.edu.co/h andle/10336/223

De Giusti, M. R. (2014). Una metodología de evaluación de repositorios digitalespara asegurar la preservación en el tiempo y el acceso a los contenidos. Tesis de Doctorado, Universidad Nacional de La Plata, La Plata. DOI: https://doi.o rg/http://sedici.unlp.edu.ar/handle/10915/43157

De Giusti, M. R. (2019). Curso de Repositorios Digitales. Universidad Nacional de La Plata. Recuperado de http://se dici.unlp.edu.ar/handle/10915/75235

De Giusti, M. R. (2020). Los repositorios institucionales: avances, desafios y nuevas prácticas. PREBI-SEDICI Universidad Nacional de La Plata. Recuperado de http://sedici.unlp.edu.ar/bitstream/handle/10915/97980/ Presentaci\%C3\%B3n.pdf-PDFA.pdf?sequence=1\&isAllowed $=\mathrm{y}$

Doria, M. V., Del Prado, A. M. \& Haustein, M. C. (2015). Repositorios digitales y software open source. Tecnología en educación \& Educación en tecnologia, 15, 73-81. Recuperado de http://sedici.unlp.edu.ar/handle/10915/46984

Eíto-Brun, R. (2014). Gestión de contenidos. Barcelona: Editorial UOC. Recuperado de https://books.google.es/books?hl=es\&lr=\&id=XzbaAwAAQBAJ\&oi=fnd\&pg=PA5\&dq=R.+Eíto-Brun. + Gestión+de+contenidos.+Vol.+17.+Editorial+UOC,

+2014\&ots=dos7H17StO\&sig=g6NZwwk9asRpFe6mXz7_Y0AiEeo

Fushimi, M. S. (2016). Acceso abierto y repositorios digitales en Argentina. En XXII Reunión de Comunicaciones Cientificas y Tecnologicas, Corrientes, Argentina. Recuperado de http://www.memoria.fahce.unlp.edu.ar/librar $\mathrm{y} ? \mathrm{a}=\mathrm{d} \& \mathrm{c}=$ eventos\&d$=\mathrm{Jev} 7116$

Fushimi, M. S. (2018, diciembre 5). Desarrollo de repositorios digitales institucionales en las universidades nacionales en Argentina, periodo 2004-2015. Recuperado de http://ridaa.unq.edu.ar/handle/20.500.11807/887

Género Bocco, M., Cruz-Lemus, J. A. y Piattini Velthuis, M. (2014). Métodos de investigación en ingeniería del software. Madrid: Editorial RaMa.

Kampffmeyer, U. (2006). Enterprise Content Management: ECM, 13-16. Recuperado de https://books.google.es/ books?hl=es\&lr=\&id=rkA-jZCdXRgC\&oi=fnd\&pg=PA17\&dq=U.+Kampffmeyer.+Enterprise+Content 
+ Management:+ECM.+Project+Consult+GmbH, $+\&$ ots $=$ lcqFmXPjvT\&sig=6QfLvzjB7HUR2Yi6p0y7tnebvTs

Kitchenham, B. A. y Pfleeger, S.L. (2008). Guide to Advanced Empirical Software Engineering, en Guide to Advanced Empirical Software Engineering. London: Springer.

Ley 25.326 (2000). Protección de los datos personales. Honorable Congreso de la Nación Argentina. Publicada en el Boletín Nacional del 02-Nov-2000. Recuperado de http://servicios.infoleg.gob.ar/infolegInternet/anexos/600 00-64999/64790/norma.htm

Ley No 26.899 (2013). Repositorios digitales institucionales de acceso abierto. Argentina. Sistema Nacional de Ciencia, Tecnología e Innovación. Publicada en el Boletín Oficial del 09-dic-2013 Recuperado de http://servicios.infoleg .gob.ar/infolegInternet/verNorma.do?id=223459

Mendes, M. A. S. \& Bax, M. P. (2018, enero 1). BPM e ECM: similaridades, diferenças e limites conceituais e tecnológicos. Transinformação, 30(1). DOI: https://doi.org/10.1590/2318-08892018000100008

Mendoza Espinoza, N. A. (2012). Construcción de un sistema de gestión de documentos internos (Memorandos) para la empresa Elecaustro S.A. de la Ciudad de Cuenca - Ecuador. Maestría en Software Libre, Universidat Oberta de Catalunya, Barcelona. Recuperado de http://openaccess.uoc.edu/webapps/o2/bitstream/10609/15078/8/nm endozaesTFC0712memoria.pdf

Molina Piñeiro, M., Marrero Sera, F. E. \& Puente Puentes, D. J. Á. (2015). Los repositorios de acceso abierto como alternativa para la visibilidad de la ciencia en las universidades: estudio de caso. Revista cubana de información en ciencias de la salud, 26(4), 330-346. Recuperado de https://www.redalyc.org/pdf/3776/377645763003.pdf

Nakano, S. \& Azrilevich, P. A. (2017). El acceso abierto y la implementación de la Ley 26.899 en la Argentina. En VII Conferencia Internacional sobre Bibliotecas y Repositorios Digitales de América Latina (BIREDIAL-ISTEC'17) y XII Simposio Internacional de Biblioteca Digitales (SIBD'17), La Plata, Argentina. Recuperado de http://sedici .unlp.edu.ar/handle/10915/63553

Nonaka, I. \& Takeuchi, H. (1995). The knowledge-creating company: how Japanese companies create the dynamics. New York: Oxford University Press.

Nuxeo, Athento. (s. f.). Recuperado de https://www.athento.com/es/nuxeo/

Nuxeo Documentation. (s. f.). Recuperado de https://doc.nuxeo.com/nxdoc/postgresql/

Polanco-Cortés, J. (2014). Repositorios digitales. Definición y pautas para su creación. San José de Costa Rica: Universidad de Costa Rica. Recuperado de https://ucrindex.ucr.ac.cr/docs/repositorios-digitales-definicion-ypautas-para-su-creacion.pdf

Porro, Y. R. \& Gálvez, L. V. (2018). Uso de la herramienta Dspace para la gestión de información: implementación de un repositorio institucional. Revista Publicando, 5(14), 329-342. Recuperado de https://revistapublicando. org/revista/index.php/crv/article/view/1280

Repositorio Institucional de la Universidad Nacional del Nordeste- RIUNNE. (s. f.). Recuperado de http://repositori o.unne.edu.ar

Sánchez M. E. \& Mariño S. I. (2020). Procedimientos de gestión documental. Una revisión preliminar de la literatura. Revista Atlante: cuadernos de educación y desarrollo. Recuperado de

Sánchez M. E., Bózzola T., Soler A. \& Mariño S. I. (2020). Metodología para el relevamiento y análisis de la información. Una propuesta para la administración central de liquidaciones en la Universidad. Ciencia latina: revista cientifica multidisciplinar, 4(1), 99-115. DOI: https://doi.org/10.37811/cl_rcm.v4i1.44

SNRD MINCYT. (s. f.). Sistema Nacional de Repositorios Digitales. Recuperado de https://repositoriosdigitales.min cyt.gob.ar

UNNE (s. f.). Universidad Nacional del Nordeste. Recuperado de https://www.unne.edu.ar/index.php?lang=es 